\title{
Linear polarization degree for detecting magnetic properties of small particles
}

\author{
Braulio García-Cámara,* Francisco González, and Fernando Moreno \\ Grupo de Óptica, Departamento Física Aplicada, Universidad de Cantabria, Avenida los Castros s/n, Santander, Spain \\ *Corresponding author: garciacb@unican.es
}

Received September 14, 2010; revised October 20, 2010; accepted October 29, 2010;

posted November 2, 2010 (Doc. ID 135066); published November 30, 2010

\begin{abstract}
Motivated by the recent advances with magnetic nanoparticles, in this research we propose a new technique for their characterization based on the measurement of certain polarimetric parameters of the scattered light, such as the linear polarization degree when it is determined at a "right-angle" scattering configuration. We will show the sensitivity of its spectral evolution with the magnetic properties of the particle. 다이 2010 Optical Society of America OCIS codes: $160.4236,290.5855$.
\end{abstract}

Current technological advances, mainly in the field of information storage, are due to the increasing interest of researchers in the magnetic properties of materials [1]. During the past few years, technological efforts have been directed to the miniaturization of data storage systems, and much research has been done on the preparation, manipulation, and characterization of magnetic nanoparticles. Several applications based on these nanoparticles have been proposed, in particular, for biomedical tasks [2] such as molecular imaging [ $\underline{3}, \underline{4}]$ or therapeutic treatments $[\underline{5}, 6]$.

At the same time, metamaterials have emerged with the objective of being able to control the optical properties of the medium, both electric and magnetic. This kind of engineered media allow us to observe magnetic permeabilities different from 1 , even in the visible range of the spectrum $[7,8]$. The possibility to manipulate both the electric permittivity and the magnetic permeability of a material is the base of several applications, such as in the field of optical communications [9-11].

One way to determine the magnetic properties of nanostructures is through the spectral characteristics of the scattered radiation: the sample is illuminated by an electromagnetic wave, and the scattered intensity is measured as a function of the incident wavelength. The analysis of certain spectral resonances can reveal magnetic responses of the scatterer. However, other techniques can be used for detecting the magnetic character of a system. In this Letter, we propose the use of polarimetric techniques and, in particular, we show the efficiency of the linear polarization degree, measured at $\theta_{\text {sca }}=90^{\circ}$ [rightangle scattering configuration (RASC)] [12], as a parameter sensitive to magnetic conducts.

The linear polarization degree $\left[P_{L}\left(\theta_{\mathrm{sca}}\right)\right]$ of the scattered intensity is defined by [13]

$$
P_{L}\left(\theta_{\text {sca }}\right)=\frac{I_{\perp}\left(\theta_{\text {sca }}\right)-I_{\|}\left(\theta_{\text {sca }}\right)}{I_{\perp}\left(\theta_{\text {sca }}\right)+I_{\|}\left(\theta_{\text {sca }}\right)}
$$

where $I_{\perp}$ and $I_{\|}$are the components of the scattered intensity linearly polarized perpendicular and parallel to the scattering plane, respectively, and are measured at the scattering angle $\theta_{\text {sca }}$.

For particles with conventional optical properties, $\mu=1$, whose size is smaller than the incident wavelength, the electric dipolar response dominates. Thus, in the far field, the angular distribution of the scattered intensity follows the typical "figure-eight shape" for the parallel component and the isotropic distribution for the perpendicular one. Consequently, $P_{L}$ measured at $\theta_{\mathrm{sca}}=90^{\circ}$ is equal to 1 , because $I_{\|}\left(90^{\circ}\right)=0$ [see Eq. (1)]. Any deviation from the dipolar behavior, such as the excitation of multipolar modes, induces changes in the distribution of the scattered intensity, which involves a decrease in the value of $P_{L}\left(90^{\circ}\right)$ [12]. On the other hand, if the scatterer is mainly magnetic and the dipolar magnetic character dominates, the angular distribution of the scattered intensity is opposite to the previous one: the isotropic distribution appears for a parallel incident polarization and the figure-eightshaped one for the perpendicular component. After checking Eq. (1), we can observe that for a dominant magnetic dipolar response, the linear polarization degree at RASC tends to be equal to -1 . Hence, we can conclude that while positive values of $P_{L}\left(90^{\circ}\right)$ are associated to an electric behavior, negative values of this polarimetric parameter correspond to a magnetic one.

To check this, we have considered a spherical particle whose optical constants are such that the scatterer presents either electric or magnetic dipolar characters at different incident frequencies. In particular, following the work of Ruppin $[\underline{14}, \underline{15}]$ :

$$
\varepsilon(\omega)=1-\frac{\omega_{p}^{2}}{\omega(\omega+i \gamma)}
$$

for the electric permittivity and

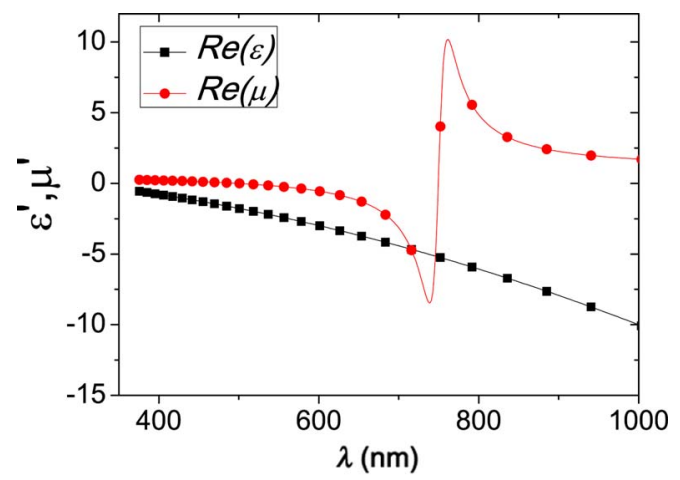

Fig. 1. (Color online) Real part of the electric permittivity (black squares) and the magnetic permeability (red circles) considered in our calculations. This material example could be left-handed between 500 and $750 \mathrm{~nm}$. 


$$
\mu(\omega)=1-\frac{F \omega^{2}}{\omega^{2}-\omega_{0}^{2}+i \omega \Gamma}
$$

for the magnetic permeability, where $\omega_{p}$ is the plasma frequency, $\omega_{0}$ is the magnetic resonance frequency, $\omega$ is the frequency of the incident electromagnetic radiation, and $\gamma$ and $\Gamma$ are damping parameters. In our numerical calculations, we have rescaled the values of [14] to the optical range in such a way that: $\omega_{p}=10 \times 10^{14} \mathrm{rad} / \mathrm{s}, \quad \omega_{0}=4 \times 10^{14} \mathrm{rad} / \mathrm{s}, \quad \gamma=0.03 \omega_{p}$, $\Gamma=0.03 \omega_{0}$, and the parameter $F=0.56$. Following these expressions, the optical constants are plotted in Fig. 1 in the considered wavelength range.

In Fig. 2, we plot the linear polarization degree measured at $90^{\circ}\left[P_{L}\left(90^{\circ}\right)-\right.$ solid curve $]$ and the extinction efficiency $\left[\left(Q_{\text {ext }}\right)-\right.$ dashed curve] of a spherical particle ( $R=100 \mathrm{~nm}$ ) as a function of the wavelength of the incident field. The considered size of the particle is such that dipolar scattering is dominant. However, a quadrupolar resonance can be observed in $Q_{\text {ext }}$ around $\lambda=750 \mathrm{~nm}$. In this case, resonances in $Q_{\text {ext }}$ are related to maximum values of Mie coefficients of the first two orders $\left(a_{1}, b_{1}\right.$, and $a_{2}$ [13] $)$, which have been shown in the figure. Also, we have plotted the angular distribution of light scattered by the particle for certain wavelengths
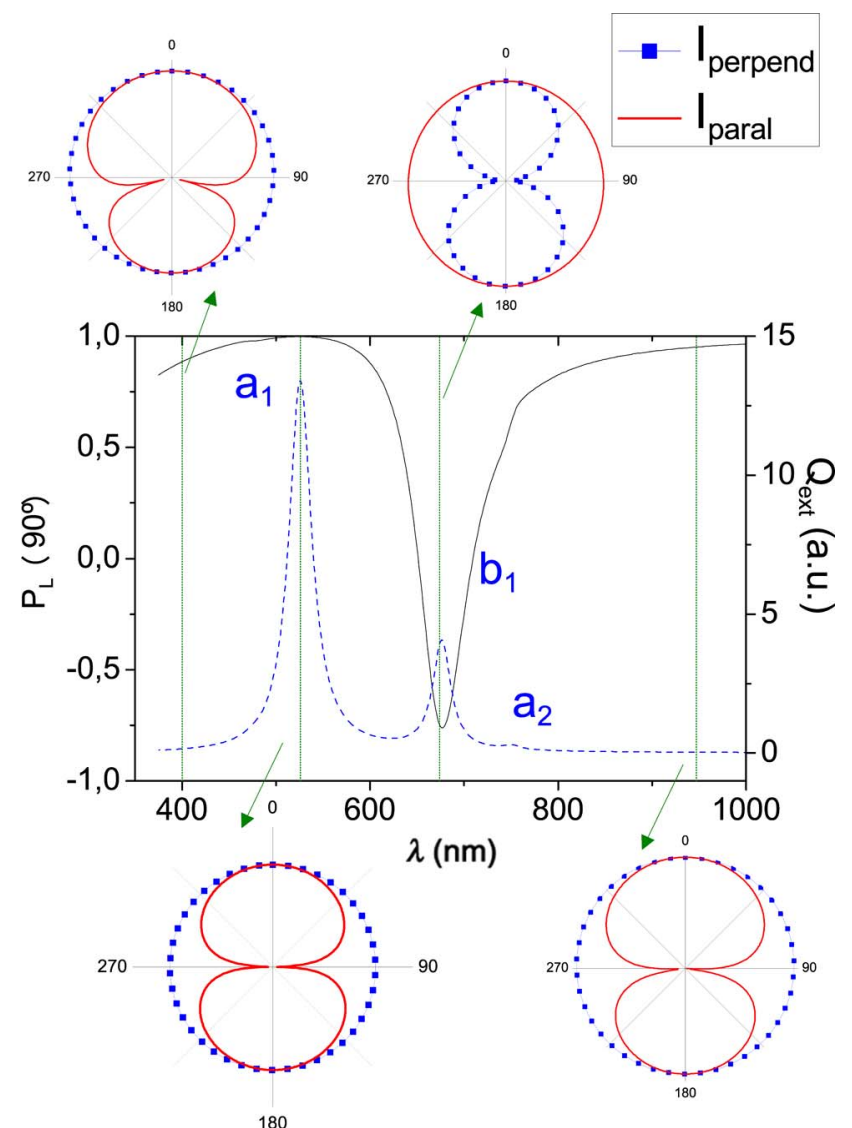

Fig. 2. (Color online) Linear polarization degree at RASC $\left(P_{L}\left(90^{\circ}\right)\right)$ [solid line] and extinction efficiency $\left(Q_{\text {ext }}\right)$ [dashed line] of a spherical particle $(R=100 \mathrm{~nm})$ as a function of the incident wavelength. Mie coefficients associated to resonances of the extinction efficiency are shown. Also, we have included the angular distribution of the scattered intensity for several cases $(\lambda=400,526,674$, and $950 \mathrm{~nm})$. of the incident electromagnetic field. Out of resonance or when the electric dipolar resonance (associated to $a_{1}$ [13]) is excited ( $\lambda<650 \mathrm{~nm}$ or $\lambda>800 \mathrm{~nm})$, the scatterer behaves like an electric dipole, as can also be seen in the scattering patterns. This means that the linear polarization degree is close to 1 . When a magnetic response dominates due to the excitation of a magnetic dipolar resonance (associated to $b_{1}$ [13]), the angular distribution reverses the shape of their components and $P_{L}\left(90^{\circ}\right)$ reaches negative values, as was predicted above. Theoretically, $P_{L}\left(90^{\circ}\right)=-1$ when the magnetic dipolar character emerges; however, in Fig. 2, the linear polarization degree reaches the value $P_{L}\left(90^{\circ}\right)=-0.8$ only because of the influence of the finite size of the particle. As the evolution of the linear polarization degree is directly related to the dipolar response of the scatterer, the influence of the particle size is crucial. In this sense, for large values of the wavelength in Fig. 2 , where the relation $R / \lambda$ reinforces the dipolar character of the scatterer, $P_{L}\left(90^{\circ}\right)$ is 1 , but for smaller $\lambda$ the linear polarization degree differs slightly from 1 .

To show the influence of particle size, in Fig. $\underline{3}$, we plot the spectral evolution of the linear polarization degree measured at RASC $\left(P_{L}\left(90^{\circ}\right)\right)$ of a spherical scatterer with four different sizes: (a) $R=100 \mathrm{~nm}$ (previously discussed in Fig. 2), (b) $R=200 \mathrm{~nm}$, (c) $R=300 \mathrm{~nm}$, and (d) $R=400 \mathrm{~nm}$. We have also included the spectral evolution of the extinction efficiency $\left(Q_{\text {ext }}\right)$ of the particles. As particle size increases [Figs. 3(b)-3(d)], other modes appear due to multipolar contributions: in particular, electric quadrupolar resonances (associated to maximum values of $a_{2}$ [13]). These resonances produce important disturbances on the angular distribution of the scattered light, which involves sharp decreases in the value of $P_{L}$ [12], such as for wavelengths around 500 and $750 \mathrm{~nm}$. However, the electric character of these resonances means that $P_{L}\left(90^{\circ}\right)$ does not reach negative values. The size also has influences on the minimum of $P_{L}$ when the magnetic resonance is excited. As can be seen, as particle
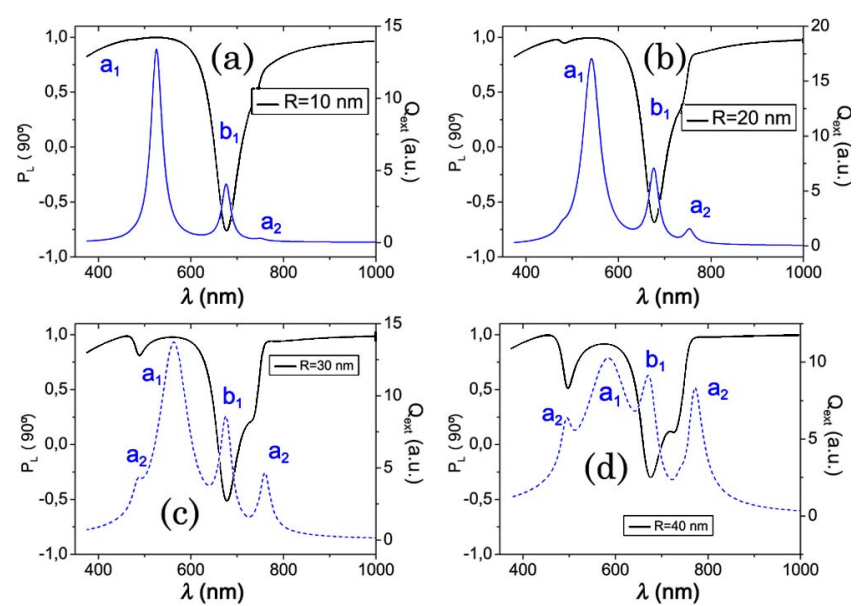

Fig. 3. (Color online) Linear polarization degree measured at RASC, $P_{L}\left(90^{\circ}\right)$ [solid curve] of a spherical particle with radius (a) $R=100 \mathrm{~nm}$, (b) $R=200 \mathrm{~nm}$, (c) $R=300 \mathrm{~nm}$, and (d) $R=400 \mathrm{~nm}$ as a function of the wavelength of the incident field. The corresponding extinction efficiencies $\left(Q_{\text {ext }}\right)$ are also plotted [dashed curve]. Mie resonances are marked with the Mie coefficients, whose maximum values are associated to each resonance. 
size increases, the value of the linear polarization degree at this point is less negative [from $P_{L}\left(90^{\circ}\right) \simeq-0.8$ for $R=$ $100 \mathrm{~nm}$ to $P_{L}\left(90^{\circ}\right) \simeq-0.3$ for $R=400 \mathrm{~nm}$.

In summary, in this research we have analyzed the possibility of using polarimetric parameters to detect magnetic responses in small particles. In particular, we have shown that the linear polarization degree at RASC remains equal to 1 when particles scatter light like an electric dipole. However, if the particle presents magnetic properties, $P_{L}\left(90^{\circ}\right)$ can get negative values. In the past few years, interest in magnetic nanoparticles has grown and this technique could be considered as an alternative method to characterize their optical properties. Also, we have checked the influence of particle size on the sensitivity of the method: as the ratio $R / \lambda$ increases, new drops in the values of $P_{L}\left(90^{\circ}\right)$ appear due to modes of order higher than the dipolar. Also, the value of the linear polarization degree when the magnetic dipolar resonance is excited is less negative. However, this can still be easily distinguished from the effect of multipolar modes.

This research has been supported by the Ministerio de Ciencia e Innovacion under project FIS2007-60158.

\section{References}

1. C. Chappert, A. Fert, and F. N. Van Dau, Nat. Mater. 6, 813 (2007).
2. J. Gao, H. Gu, and B. Xu, Acc. Chem. Res. 42, 1097 (2009).

3. J.-H. Lee, Y.-M. Huh, Y.-W. Jun, J.-W. Seo, J.-T. Jang, H.-T. Song, S. Kim, E.-J. Cho, H.-G. Yoon, J.-S. Suh, and J. Cheon, Nat. Med. 13, 95 (2006).

4. R. Bardhan, W. Chen, C. Perez-Torres, M. Bartels, R. M. Huschka, L. L. Zhao, E. Morosan, R. G. Pautler, A. Joshi, and N. J. Halas, Adv. Funt. Mat. 19, 3901 (2009).

5. C. Alexiou, R. J. Schmid, R. Jurgons, M. Kremer, G. Wanner, C. Bergemann, E. Huenges, T. Nawroth, W. Arnold, and F. G. Parak, Eur. Biophys. J. 35, 446 (2006).

6. J. R. McCarthy and R. Weissleder, Adv. Drug Deliv. Rev. 60, 1241 (2008).

7. V. M. Shalaev, Nat. Photon. 1, 41 (2007).

8. C. Enkrich, M. Wegener, S. Linden, S. Burger, L. Zschiedrich, F. Schmidt, J. F. Zhou, Th. Koschny, and C. M. Soukoulis, Phys. Rev. Lett. 95, 203901 (2005).

9. B. Kante, A. Ourir, S. N. Burokur, F. Gadot, and A. de Lustrac, C. R. Phys. 9, 31 (2008).

10. D. A. Miller, Nat. Photon. 4, 3 (2010).

11. B. García-Cámara, J. M. Saiz, F. González, and F. Moreno, Metamaterials 4, 15 (2010).

12. B. Setién, P. Albella, J. M. Saiz, F. González, and F. Moreno, New J. Phys. 12, 103031 (2010).

13. C. F. Bohren and D. R. Huffman, Absorption and Scattering Light by Small Particles (Wiley, 1983).

14. R. Ruppin, Sol. State Commun. 116, 411 (2000).

15. R. Ruppin and O. J. F. Martin, J. Chem. Phys. 121, 11358 (2004). 